\title{
Ultrasound evaluation for prediction of outcomes and surgical decision in fetal hydronephrosis
}

\author{
DAN ZHANG ${ }^{1}$, XIELU SUN $^{1}$, XIAOLE CHEN ${ }^{1}$, BEIBEI YU ${ }^{1}$, TIANRAN LI ${ }^{1}$, YUEYUE CHENG ${ }^{1}$, \\ MANJING YE ${ }^{1}$, LINGLING LIN ${ }^{1}$, LIE MA ${ }^{1}$, YAPING ZHAO ${ }^{1}$ and PIHONG LI $^{2}$ \\ Departments of ${ }^{1}$ Ultrasonography and ${ }^{2}$ General Surgery, The Second Affiliated Hospital of \\ Wenzhou Medical University, Wenzhou, Zhejiang 325027, P.R. China
}

Received January 6, 2019; Accepted April 23, 2019

DOI: $10.3892 /$ etm.2019.7683

\begin{abstract}
Fetal hydronephrosis (HY) is a frequent congenital condition, which may be detected by prenatal ultrasound. Society for Fetal Urology (SFU) and anterior-posterior diameter (APD) grading are two major grading systems based on ultrasonography. The present study aimed to assess the predictive value of the SFU and APD grades in patients with fetal HY. A total of 162 patients with 234 kidneys affected by HY were included in the present study. The SFU and APD grades were determined from the ultrasound images at 38 gestational weeks, and a 12-month follow-up was performed after birth. The associations of the SFU and APD grades with the outcome of fetal HY, including HY regression, and post-partum surgery were examined. In the present study, 16 patients with 17 kidneys were diagnosed with pathological HY, and stenosis at the ureteropelvic junction was demonstrated to be a leading cause of pathological HY. Among the 234 kidneys affected by HY, 161 kidneys were scored as SFU grade I, 57 as SFU grade II, 7 as SFU grade III and 9 kidneys as SFU grade IV. According to the APD grading system, 112 kidneys were determined as having low, 104 as having moderate and 18 as having severe HY. The SFU and APD grades were demonstrated to be independently associated with the occurrence of pathological HY by logistic regression analysis with a high diagnostic accuracy to distinguish pathological and physiological HY cases as evidenced by the results of ROC analysis. In addition, univariate and
\end{abstract}

Correspondence to: Dr Yaping Zhao, Department of Ultrasonography, The Second Affiliated Hospital of Wenzhou Medical University, 109 West Xueyuan Road, Wenzhou, Zhejiang 325027, P.R. China

E-mail: zhongguo222706@163.com

Dr Pihong Li, Department of General Surgery, The Second Affiliated Hospital of Wenzhou Medical University, 109 West Xueyuan Road, Wenzhou, Zhejiang 325027, P.R. China

E-mail: lipihongwz@163.com

Key words: ultrasound, Society for Fetal Urology, anterior-posterior diameter, hydronephrosis, surgery multivariate logistic regression analysis indicated that patients with spontaneous HY regression usually had low SFU and APD grades. Furthermore, the rate of surgery was increased in the group of patients with high SFU or APD grades, and these two systems were identified as independent predictors for the requirement of surgery by Kaplan-Meier analysis. Patients with pathological HY had high SFU and APD grades, and these two grading systems may be used as reliable predictors for the outcome of fetal HY, including HY regression, and post-partum surgery.

\section{Introduction}

Fetal hydronephrosis (HY), a type of fetal malformation, is a common congenital condition (1). It may be determined by prenatal ultrasound and is characterized by abnormal dilatation of renal pelvis and calyces, as well as atrophy in renal parenchyma $(2,3)$. The incidence rate of HY among all newborns detected by ultrasound is $0.6-5.4 \%$ (4). HY is more frequently diagnosed in fetuses of male gender and those in a unilateral position (5). Studies have demonstrated that vesicoureteral reflux and tract obstruction, particularly at the ureteropelvic junction, are the leading causes of HY, while HY caused by duplex renal system or hydroureteronephrosis is rare $(6,7)$. HY may be divided into physiological and pathological types based on the post-partum conditions. HY that may spontaneously resolve after birth is classified as physiological HY, while the type that deteriorates and may lead to impaired renal function is referred to as pathological HY (8).

At present, fetal HY is mainly diagnosed based on prenatal ultrasound (9). The rate of clinical detection of HY has been increasing due to the development of ultrasonic technology and optimization of ultrasonic instruments (10). However, a dilemma is emerging with regard to the management of infants diagnosed with fetal HY $(11,12)$. Society for Fetal Urology (SFU) grading and anterior-posterior diameter (APD) measurement are two major ultrasonography-based systems to evaluate HY. The SFU system consists of five grades (0, I, II, III and IV) according to the appearance of calices, pelvis and thinning of parenchyma (13). The APD system contains three grades (mild, moderate and severe) based on the anterior/posterior diameter of the pelvis (14). The severity of HY evaluated by ultrasound is closely associated with the prognosis of the 
affected pediatric patients. Thus, precise determination of $\mathrm{HY}$ grading is critical for optimal management.

To further investigate the diagnosis and achieve appropriate management of fetal HY, the present study focused on the predictive value of the SFU and APD grading systems regarding the post-partum outcome, and explored their associations with spontaneous regression of HY and requirement of surgery.

\section{Materials and methods}

Subjects. The present study retrospectively analyzed the ultrasonic data and clinical characteristics of 162 pregnant females who received antenatal examination and gave birth at the Second Affiliated Hospital of Wenzhou Medical University (Wenzhou, China) between January 2014 and December 2017. All of these subjects fulfilled the inclusion criteria: Singleton pregnancy; detection of fetal HY by ultrasound; no fetal malformation except HY; no consanguineous marriage; couples had no genetic disease. Prenatal ultrasound of these pregnant females at 20-40 gestational weeks identified 234 fetal kidneys affected by HY, including 72 cases of bilateral HY and 90 of unilateral HY. The protocols of the present study were approved by the Ethics Committee of the Second Affiliated Hospital of Wenzhou Medical University (Wenzhou, China) and written informed consent for the use of ultrasonic data and clinical characteristics was obtained from each participant.

Imaging. All of the pregnant females underwent assessment of fetal HY by ultrasonography with an ESAOTE Technos MYLAB 65 (Esaote) every 2-4 weeks prior to delivery. The neonates were re-examined by ultrasound at 7 days after delivery. The cases still exhibiting HY were followed up for 12 months, and those patients with persistent or progressive HY were further diagnosed by other adjuvant examinations, including intravenous pyelography (IVP), emission computed tomography (ECT) and magnetic resonance urography (MRU). An X-ray machine (Siemens AG, Munich, Germany) was used for IVP, a CT scanner (Siemens AG) was used for ECT, and a nuclear magnetic resonance detector (Philips NV, Amsterdam, Netherlands) was used for MRU examination. HY that spontaneously resolves after birth is referred to as physiological $\mathrm{HY}$, while the type that becomes deteriorative and may lead to impaired renal function is called pathological HY. During the follow-up survey, the HY outcomes and rate of surgery were recorded for further analysis. The patients were graded using the SFU and APD systems based on their ultrasound images (15). The classifications of these two systems are listed in Table I and representative ultrasound images are provided in Fig. 1.

Statistical analysis. All the statistical analyses were performed using SPSS 20.0 software (IBM Corp.). Continuous variables are expressed as the mean \pm standard deviation. The association between SFU or APD grades and the clinical characteristics of the patients was assessed using the Chi-squared test. Univariate and multivariate logistic regression analysis was performed to examine the association of clinicopathological parameters, as well as SFU or APD grading, with the postpartum outcomes, HY regression and post-partum surgery
Table I. Classification criteria of SFU and APD grading systems.

\begin{tabular}{ll} 
A, SFU grading system & \\
\hline Grade & \multicolumn{1}{c}{ Characteristics } \\
\hline 0 & $\begin{array}{l}\text { No hydronephrosis } \\
\text { Renal pelvis is slightly separated } \\
\text { I }\end{array}$ \\
II & $\begin{array}{l}\text { Renal pelvis is further separated } \\
\text { and a single or a few dilated } \\
\end{array}$ \\
calices may be visualized \\
III & $\begin{array}{l}\text { All calices are dilated } \\
\text { All calices are dilated and the } \\
\text { IV }\end{array}$ \\
& $\begin{array}{l}\text { renal parenchyma over the } \\
\text { calices is thinned }\end{array}$ \\
\hline
\end{tabular}

$\mathrm{B}, \mathrm{APD}$ grading system

\begin{tabular}{ll}
\hline Grade & \multicolumn{1}{c}{ Time window/APD $(\mathrm{mm})$} \\
\hline Mild & Second trimester: $4<$ APD $<7$ \\
& Third trimester: $7<$ APD $<9$ \\
Moderate & Second trimester: $7<$ APD $<10$ \\
& Third trimester: $9<$ APD $<15$ \\
Severe & Second trimester: APD $>10$ \\
& Third trimester: APD $>15$ \\
\hline
\end{tabular}

SFU, Society for Fetal Urology; APD, anterior-posterior diameter.

for fetal HY. Receiver operating characteristic (ROC) curve analysis was performed to evaluate the predictive value of SFU and APD grading systems, and the sensitivity and specificity to distinguish pathological and physiological HY was determined. The Kaplan-Meier method was applied to analyze the rate of surgery among the patients with pathological HY and different SFU and APD grades, and log-rank test was adopted to compare the differences between curves. $\mathrm{P}<0.05$ was considered to indicate statistical significance.

\section{Results}

Characteristics of the patients and grading results. The characteristics of the patients are summarized in Table II. According to the prenatal ultrasound, a total of 234 kidneys were affected by fetal HY, including 60 cases with the left and 30 cases with the right kidney affected, as well as 72 cases with bilateral involvement. According to the post-partum examination, 217 kidneys $(92.7 \%$; $n=146)$ had physiological HY and 17 kidneys (7.3\%; n=16) had pathological HY.

SFU and APD grading were performed based on the ultrasound images at 38 gestational weeks. A total of 161 kidneys were scored as SFU grade I, 57 kidneys were SFU grade II, 7 kidneys were SFU grade III and 9 kidneys were SFU grade IV. According to the APD grading system, 112 kidneys were determined as having mild, 104 kidneys as moderate and 18 kidneys as displaying severe HY (Table II). 
Table II. Characteristics and grading results of the HY patients.

\begin{tabular}{lcc}
\hline Parameters & $\begin{array}{c}\text { No. of patients }(\%) \\
\text { or mean } \pm \text { SD (range) }\end{array}$ & $\begin{array}{c}\text { No. of } \\
\text { kidneys }(\%)\end{array}$ \\
\hline Gender & & \\
Female & $64(39.5)$ & $91(38.9)$ \\
Male & $98(60.5)$ & $143(61.1)$ \\
Affected side & & $60(25.6)$ \\
Left & $60(37.0)$ & $30(12.8)$ \\
Right & $30(18.5)$ & $144(61.5)$ \\
Bilateral & $72(44.4)$ & \\
Maternal age (years) & $29.8 \pm 5.2(20-41)$ & $17(7.3)$ \\
Post-partum outcome & & $161(68.8)$ \\
Physiological HY & $146(90.1)$ & $57(2.4)$ \\
Pathological HY & $16(9.9)$ & $7(3.0)$ \\
SFU grade & & $9(3.8)$ \\
I & $111(68.5)$ & $112(47.9)$ \\
II & $36(22.2)$ & $(44.4)$ \\
III & $7(4.3)$ & \\
IV & $8(4.9)$ & \\
APD grade & & \\
Mild & $72(44.4)$ & $(10.5)$ \\
Moderate & & \\
Severe & & \\
\hline
\end{tabular}

HY, hydronephrosis; SFU, Society for Fetal Urology; APD, anterior-posterior diameter; SD, standard deviation.

Association of SFU and APD grades with the clinicopathological characteristics of the patients. To examine the association of clinical characteristics with SFU and APD grades, the Chi-squared test was first adopted, indicating that the patients' gender, affected side or maternal age were not significantly associated with the SFU or APD grade (all P $>0.05$; Table III). By contrast, the SFU and APD grade were associated with the post-partum outcome (all $\mathrm{P}<0.001)$.

SFU and APD grades are independently associated with the post-partum outcome of fetal HY. Logistic regression analysis was then performed to assess the influence of clinicopathological features, and the SFU and APD grading results of the patients on post-partum outcome of fetal HY (Table IV). The univariate analysis revealed that an SFU grade of III/IV and severe HY according to the APD were significantly associated with pathological HY, which indicated that the pathological HY cases were more frequently SFU grade III/IV $(\mathrm{P}<0.001)$ and had a larger APD $(\mathrm{P}<0.001)$. The multivariate analysis then suggested that the SFU grade $(\mathrm{OR}=177.840,95 \%$ $\mathrm{CI}=16.628-1902.081, \mathrm{P}<0.001)$ and APD grade $(\mathrm{OR}=28.209$, 95\% CI=4.230-196.218, $\mathrm{P}=0.001$ ) were independently associated with the occurrence of pathological HY.

Diagnostic accuracy of SFU and APD grading systems to distinguish pathological from physiological $H Y$. Considering the close association of the SFU and APD grading systems
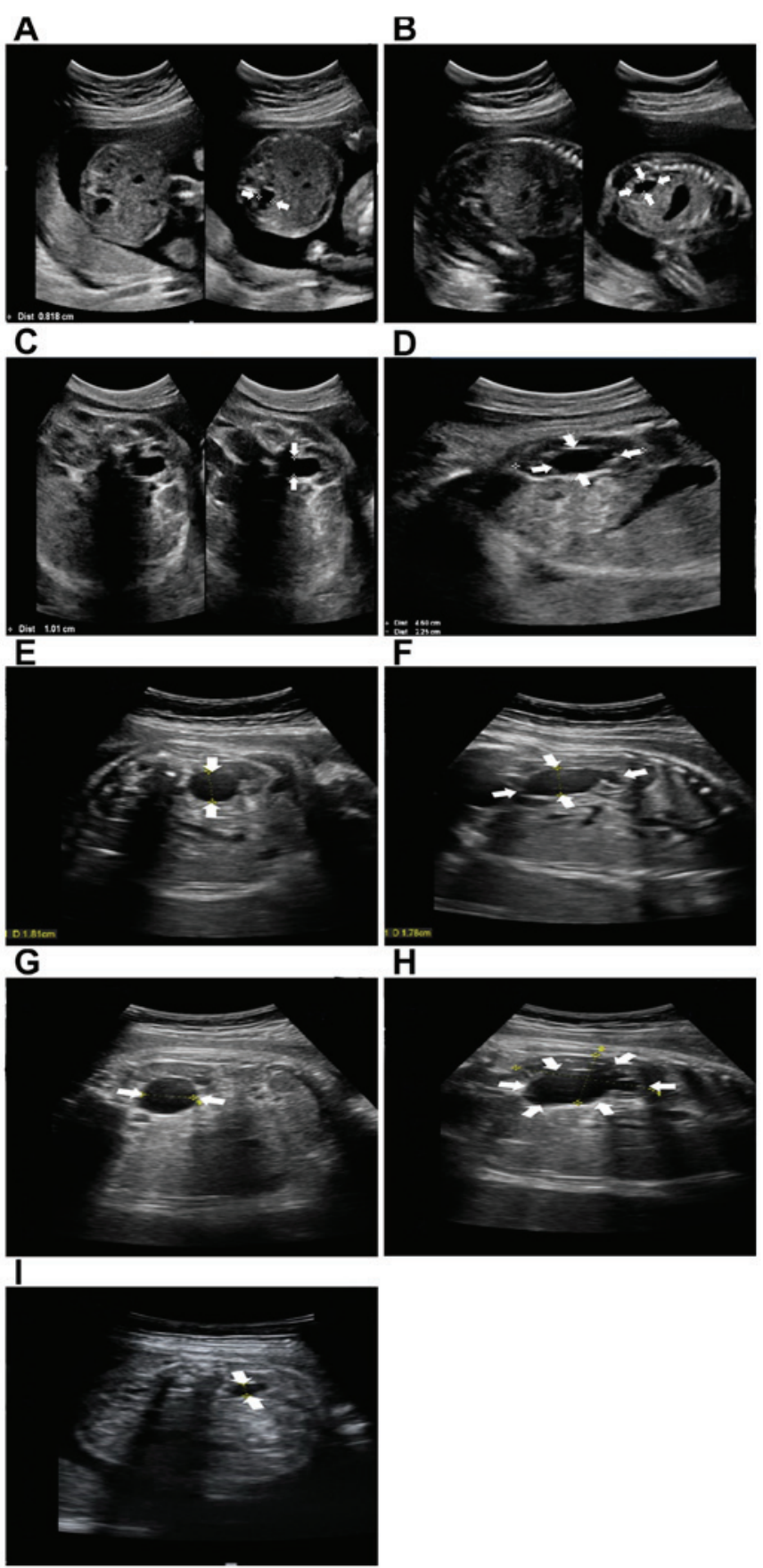

Figure 1. Ultrasound images of subjects with hydronephrosis with different SFU and APD grades. (A) Cross section of kidney with SFU grade I. (B) Vertical section of kidney with SFU grade I. (C) Cross section of kidney with SFU grade II. (D) Vertical section of kidney with SFU grade II. (E) Cross section of kidney with SFU grade III. (F) Vertical section of kidney with SFU grade III. (G) Cross section of kidney with SFU grade IV. (H) Vertical section of kidney with SFU grade IV. (I) Standard measuring section of APD. White arrows indicate the dilatation of the renal pelvis. SFU, Society for Fetal Urology; APD, anterior-posterior diameter.

with the post-partum outcome of fetal HY, their diagnostic value was further evaluated to with regard to their ability to distinguish patients with pathological HY from those with physiological HY. The sensitivity and specificity of the SFU grading system was 70.6 and $98.2 \%$, respectively, and the Youden index (YI) was 0.688 for the discrimination of patients with pathological HY from the cases of 
Table III. Association of SFU and APD grades with clinical characteristics of the HY patients.

\begin{tabular}{|c|c|c|c|c|c|c|c|}
\hline \multirow[b]{2}{*}{ Features } & \multirow[b]{2}{*}{$\begin{array}{l}\text { Affected kidneys } \\
\qquad(\mathrm{n}=234)\end{array}$} & \multicolumn{2}{|c|}{ SFU grade } & \multirow[b]{2}{*}{ P-value } & \multicolumn{2}{|c|}{ APD grade } & \multirow[b]{2}{*}{ P-value } \\
\hline & & $\begin{array}{c}\mathrm{I} / \mathrm{II} \\
(\mathrm{n}=218)\end{array}$ & $\begin{array}{l}\text { III/IV } \\
(\mathrm{n}=16)\end{array}$ & & $\begin{array}{l}\text { Mild/moderate } \\
\quad(n=216)\end{array}$ & $\begin{array}{l}\text { Severe } \\
(n=18)\end{array}$ & \\
\hline Gender & & & & 0.238 & & & 0.314 \\
\hline Female & 91 & 87 & 4 & & 86 & 5 & \\
\hline Male & 143 & 131 & 12 & & 130 & 13 & \\
\hline Affected side & & & & 0.290 & & & 0.269 \\
\hline Left & 132 & 125 & 7 & & 125 & 8 & \\
\hline Right & 102 & 93 & 9 & & 91 & 10 & \\
\hline Maternal age (years) & & & & 0.120 & & & 0.111 \\
\hline$\leq 30$ & 145 & 138 & 7 & & 137 & 8 & \\
\hline$>30$ & 89 & 80 & 9 & & 79 & 10 & \\
\hline Post-partum outcome & & & & $<0.001$ & & & $<0.001$ \\
\hline Physiological HY & 217 & 213 & 4 & & 211 & 6 & \\
\hline Pathological HY & 17 & 5 & 12 & & 5 & 12 & \\
\hline
\end{tabular}

Data in this table represents number of kidneys. P-values in the table were obtained using the Chi-square test. HY, hydronephrosis; SFU, Society for Fetal Urology; APD, anterior-posterior diameter.

Table IV. Univariate and multivariate logistic regression analysis of the association of clinicopathological features with pathological fetal HY.

\begin{tabular}{|c|c|c|c|c|c|c|}
\hline \multirow[b]{2}{*}{ Parameters } & \multicolumn{3}{|c|}{ Univariate analysis } & \multicolumn{3}{|c|}{ Multivariate analysis } \\
\hline & OR & $95 \% \mathrm{CI}$ & P-value & OR & $95 \% \mathrm{CI}$ & P-value \\
\hline Gender (male vs. female) & 1.181 & $0.421-3.311$ & 0.752 & 0.183 & $0.024-1.404$ & 0.102 \\
\hline Affected side (left vs. right) & 0.899 & $0.330-2.450$ & 0.835 & 1.783 & $0.284-11.195$ & 0.537 \\
\hline Maternal age ( $>30$ vs. $\leq 30$ years) & 2.459 & $0.914-6.815$ & 0.074 & 2.307 & $0.392-13.567$ & 0.355 \\
\hline SFU grade (III/IV vs. I/II) & 127.800 & $30.351-538.134$ & $<0.001$ & 177.840 & $16.628-1902.081$ & $<0.001$ \\
\hline APD grade (severe vs. mild/moderate) & 64.472 & $17.859-232.746$ & $<0.001$ & 28.209 & $4.230-196.218$ & 0.001 \\
\hline
\end{tabular}

HY, hydronephrosis; SFU, Society for Fetal Urology; APD, anterior-posterior diameter: OR, odds ratio.

physiological HY. The sensitivity and specificity of the APD grading system was 70.6 and $97.2 \%$, respectively, with a YI of 0.678 (Table V). In addition, the ROC curves for these two systems were constructed, and the area under the curve (AUC) was 0.958 for the SFU grade and 0.915 for the APD grade (Fig. 2). These results indicated that the SFU and APD grading systems have a relatively high diagnostic accuracy. Furthermore, we the diagnostic performance of the combined SFU and APD systems was evaluated, resulting in an AUC of 0.986 , a sensitivity of $82.4 \%$, a specificity of $98.6 \%$ and a YI of 0.810 , suggesting that the combination of the SFU and APD grading systems has a higher diagnostic value compared with that of these two systems alone regarding the discrimination of cases of pathological HY from those of physiological HY.

Influence of SFU and APD grades on $H Y$ regression. The influence of the SFU and APD grades on fetal HY regression was then assessed. The 234 kidneys affected by HY included 204 kidneys with spontaneously resolved HY (87.2\%) and 30 with persistent HY (12.8\%). As presented in Table VI, univariate logistic regression analysis indicated that an SFU grade of I/II and mild to moderate HY according to APD grading are associated with HY regression (all $\mathrm{P}<0.001$ ), suggesting that those cases with resolution of HY more frequently had low SFU and APD grades. Furthermore, multivariate logistic regression analysis revealed that the $\mathrm{SFU}$ grade $(\mathrm{OR}=24.843$, 95\% $\mathrm{CI}=5.471-112.804, \mathrm{P}<0.001)$ and $\mathrm{APD}$ grade $(\mathrm{OR}=21.301$, $95 \% \mathrm{CI}=5.398-84.047, \mathrm{P}<0.001)$ were independently associated with the occurrence of $\mathrm{HY}$ regression.

Association of SFU and APD grades with the rate of surgery. To facilitate optimal management of HY patients, further analysis focused on the association of SFU and APD grades with post-partum surgery. Among the 16 patients with pathological HY, 12 cases received surgery. As presented in Fig. 3, 
Table V. Diagnostic accuracy of SFU and APD grading systems to distinguish pathological and physiological HY.

\begin{tabular}{|c|c|c|c|c|c|}
\hline Parameters & Physiological HY & Pathological HY & Sensitivity & Specificity & YI \\
\hline SFU grade & & & $70.6 \%$ & $98.2 \%$ & 0.688 \\
\hline I-II & 213 & 5 & & & \\
\hline III-IV & 4 & 12 & & & \\
\hline APD grade & & & $70.6 \%$ & $97.2 \%$ & 0.678 \\
\hline Mild-moderate & 211 & 5 & & & \\
\hline Severe & 6 & 12 & & & \\
\hline SFU grade + APD grade & & & $82.4 \%$ & $98.6 \%$ & 0.810 \\
\hline I-II + Mild-moderate & 214 & 3 & & & \\
\hline III-IV + Severe & 3 & 14 & & & \\
\hline
\end{tabular}

HY, hydronephrosis; SFU, society for Fetal Urology; APD, anterior-posterior diameter; YI, youden index.
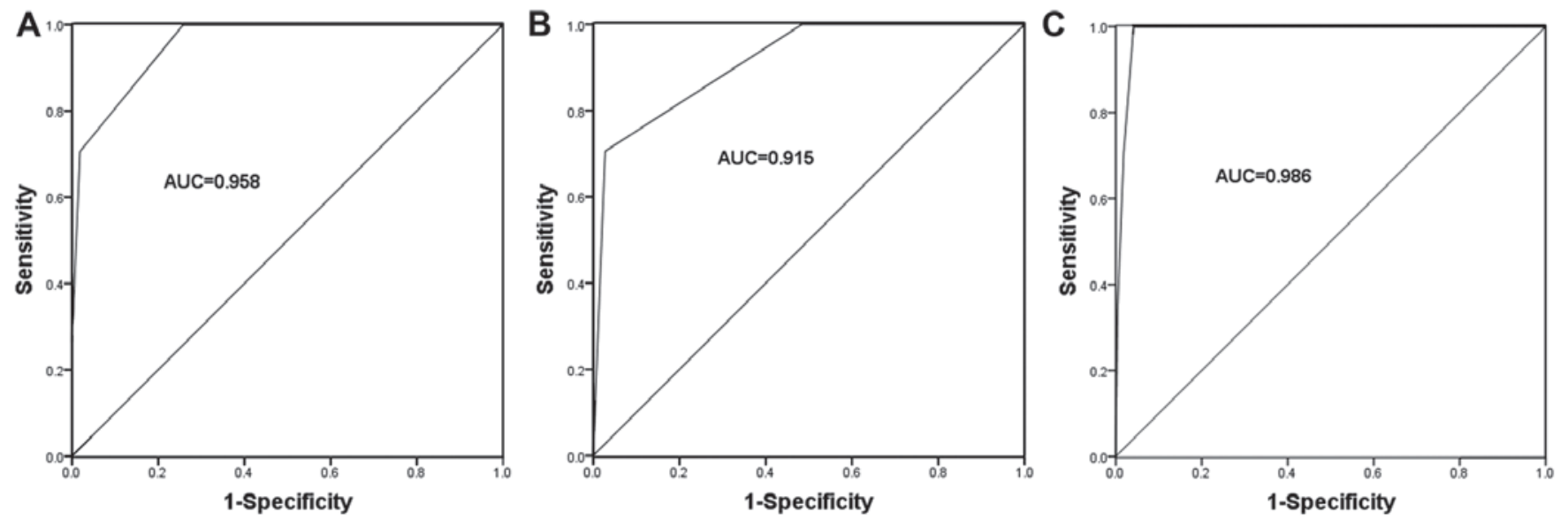

Figure 2. ROC curves for fetal hydronephrosis patients based on SFU and APD grading systems. (A) The SFU grade had high diagnostic accuracy with an AUC of 0.958. (B) The APD grade had a high diagnostic accuracy with an AUC of 0.915. (C) Combination of SFU and APD grading system had an enhanced diagnostic accuracy with an AUC of 0.986. SFU, Society for Fetal Urology; APD, anterior-posterior diameter; AUC, area under the ROC curve; ROC, receiver operating characteristic.

those patients with a high SFU or APD grade had a higher surgery rate compared with those with a low SFU (log-rank $\mathrm{P}=0.007$ ) or APD grade (log-rank $\mathrm{P}=0.008)$. Furthermore, univariate logistic regression analysis suggested that an SFU grade III/IV and severe HY according to APD grading were associated with a high surgery rate (all $\mathrm{P}<0.001)$. Multivariate logistic regression analysis further confirmed that the SFU $(\mathrm{OR}=55.973,95 \% \mathrm{CI}=2.202-1422.788, \mathrm{P}=0.015)$ and $\mathrm{APD}$ grades $(\mathrm{OR}=31.365,95 \% \mathrm{CI}=1.306-753.495$, $\mathrm{P}=0.034)$ were independent indicators for the occurrence of surgery (Table VII).

Characteristics of the patients diagnosed with pathological $H Y$. The characteristics of the patients with pathological HY are summarized in Table VIII. The patients who received post-partum surgery had a good prognosis, as their HY was resolved following the surgery. However, the prognosis of the patients without surgery was unclear due to no available related follow-up data. The etiological data regarding pathological HY indicated that stenosis at the ureteropelvic junction was the leading cause of pathological fetal HY.

\section{Discussion}

Fetal HY refers to the dilatation and separation of the fetal renal pelvis system caused by the obstruction of urine excretion from the kidney (16). It is generally considered that HY is a temporary clinical manifestation of fetal urinary system dysfunction (17). In the majority of cases, fetal HY spontaneously resolves with the increase of gestational weeks or after birth, and is then defined as physiological HY, while persistent or aggravated HY are defined as pathological HY. Woodward and Frank (18) indicated that reversible HY, which regresses during fetal growth and development, accounted for $\sim 65 \%$ of all fetal HY cases. A total of 162 patients were included in the present study, with 234 kidneys diagnosed with fetal HY. A total of 16 patients with 17 kidneys were determined to have pathological fetal HY, accounting for $9.9 \%$ of all HY patients, and the HY in the remaining cases regressed after birth. Previous studies revealed that pathological HY is mainly caused by structural abnormalities of the renal pelvis system and urine reflux $(6,19)$. The present study indicated that 9 patients, accounting for $56.3 \%$ of all pathological HY 
Table VI. Univariate and multivariate logistic regression analysis of the influence of clinicopathological features on HY regression.

\begin{tabular}{lrrrrrrrr}
\hline & \multicolumn{3}{c}{ Univariate analysis } & & \multicolumn{3}{c}{ Multivariate analysis } \\
\cline { 2 - 3 } Parameters & OR & $95 \% \mathrm{CI}$ & P-value & & OR & $95 \%$ CI & P-value \\
\hline Gender (male vs. female) & 0.948 & $0.433-2.074$ & & 0.894 & & 0.877 & $0.318-2.422$ & 0.800 \\
Affected side (left vs. right) & 1.153 & $0.535-2.488$ & & 0.716 & & 1.085 & $0.389-3.024$ & 0.876 \\
Maternal age ( $>30$ vs. $\leq 30$ years) & 2.810 & $1.281-6.162$ & & 0.010 & & 2.585 & $0.913-7.315$ & 0.074 \\
SFU grade (III/IV vs. I/II) & 51.235 & $13.289-197.531$ & $<0.001$ & & 24.843 & $5.471-112.804$ & $<0.001$ \\
APD grade (severe vs. mild/moderate) & 30.435 & $9.694-95.559$ & $<0.001$ & & 21.301 & & $5.398-84.047$ & $<0.001$ \\
\hline
\end{tabular}

HY, hydronephrosis; SFU, Society for Fetal Urology; APD, anterior-posterior diameter; OR, odds ratio.
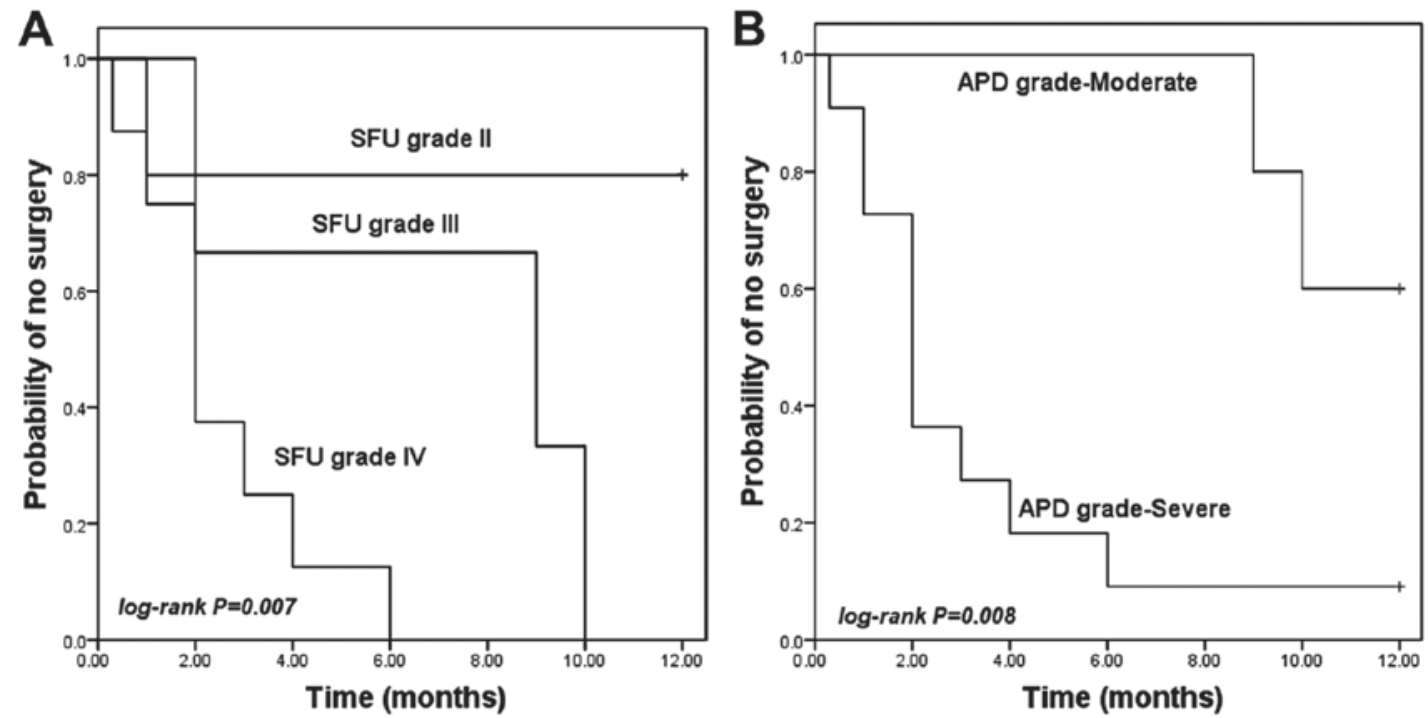

Figure 3. Association of SFU or APD grades with the post-partum surgery rate evaluated using the Kaplan-Meier method. (A) Patients with higher SFU grades had a higher surgery rate compared with those with lower SFU grades (log-rank $\mathrm{P}=0.007$ for all three curves). (B) Severe hydronephrosis ( $\mathrm{n}=12$ ) according to the APD grade was associated with a high rate of surgery (log-rank $\mathrm{P}=0.008)$. SFU, Society for Fetal Urology; APD, anterior-posterior diameter.

cases, had stenosis at the ureteropelvic junction, which was the leading cause of pathological HY in this cohort.

Prenatal ultrasound is a widely used method to monitor fetal HY. The SFU and APD grading systems are the two most frequently used standardized systems for the evaluation of HY based on ultrasound images (20). The qualitative assessment of HY via the SFU grading system is based on the degree of pelvicaliectasis and cortical thinning. However, the determination of certain non-quantitative features is limited by inter-observer variability (21). The APD grading system is used to determine the degree of pelviectasis, which mainly relies on the greatest diameter of the renal pelvis measured in the ultrasound images (22). To further facilitate the diagnosis and appropriate management of fetal HY, the present study sought to investigate the predictive value of SFU and APD grades for the post-partum outcome, and explore their association with spontaneous regression of HY and requirement for surgery.

The present study first assessed the association of the SFU and APD grades with the occurrence of pathological HY. The results indicated that the kidneys with pathological HY usually had a high SFU and APD grade, and the grades according to these two systems were identified as two independent indicators for the presence of pathological HY. Furthermore, the present study assessed whether these two systems may be used to distinguish cases of pathological HY from those with physiological HY. Thus, the YI was calculated and a ROC analysis was performed, revealing that the SFU and APD grading systems had a high diagnostic accuracy for pathological HY. Of note, combination of the SFU and APD grading systems increased the diagnostic accuracy as compared with that of each grading system alone. These results suggested that the combined method provides a reliable predictive tool for the outcome of fetal HY.

Although the incidence of detected fetal HY has increased in recent decades, the condition spontaneously resolves in most cases after birth (23). The present study performed a 12-month post-partum follow-up, during which HY was identified to be resolved in 204 kidneys, accounting for $87.2 \%$ of all HY cases. The association of SFU and APD grades with the occurrence of HY regression was further assessed by univariate and multivariate linear regression analyses using the data at 38 gestational weeks. Most of the resolved HY cases had low SFU or APD grades, and the SFU and APD grading systems were 
Table VII. Univariate and multivariate logistic regression analysis of the influence of clinicopathological features on the requirement of surgery.

\begin{tabular}{|c|c|c|c|c|c|c|}
\hline \multirow[b]{2}{*}{ Parameters } & \multicolumn{3}{|c|}{ Univariate analysis } & \multicolumn{3}{|c|}{ Multivariate analysis } \\
\hline & OR & $95 \% \mathrm{CI}$ & P-value & OR & $95 \% \mathrm{CI}$ & P-value \\
\hline Gender (male vs. female) & 1.019 & $0.323-3.218$ & 0.974 & 0.833 & $0.067-10.338$ & 0.887 \\
\hline Affected side (left vs. right) & 1.116 & $0.363-3.429$ & 0.848 & 1.828 & $0.138-24.212$ & 0.647 \\
\hline Maternal age ( $>30$ vs. $\leq 30$ years) & 2.765 & $0.875-8.737$ & 0.083 & 2.265 & $0.212-24.239$ & 0.499 \\
\hline SFU grade (III/IV vs. I/II) & 651.000 & $67.453-6282.911$ & $<0.001$ & 55.973 & $2.202-1422.788$ & 0.015 \\
\hline APD grade (severe vs. mild/moderate) & 430.000 & $47.866-3862.866$ & $<0.001$ & 31.365 & $1.306-753.495$ & 0.034 \\
\hline
\end{tabular}

SFU, Society for Fetal Urology; APD, anterior-posterior diameter; OR, odds ratio.

Table VIII. Characteristics of the patients diagnosed with pathological hydronephrosis.

\begin{tabular}{lllcc}
\hline SFU grade & APD grade & \multicolumn{1}{c}{ Etiology } & Surgery & Prognosis after surgery \\
\hline IV & Severe & Stenosis at the left ureteropelvic junction & Yes & Favorable \\
IV & Severe & Obstruction at the left middle ureter & Yes & Favorable \\
IV & Severe & Stenosis at the right ureteropelvic junction & Yes & Favorable \\
II & Severe & Stenosis at the left ureteropelvic junction & Yes & Favorable \\
IV & Severe & Stenosis at the left ureteropelvic junction & Yes & Favorable \\
IV & Severe & Stenosis at the left ureteropelvic junction & Yes & Favorable \\
III & Moderate & Stenosis at the end of left ureter & Yes & Favorable \\
IV & Severe & Stenosis at the left ureteropelvic junction & Yes & Favorable \\
III & Severe & Stenosis at the left ureteropelvic junction & Yes & Favorable \\
IV & Severe & Stenosis at the end of left ureter & Yes & Favorable \\
Left: IV; & Left: Severe; & Posterior urethral valve & Yes & Favorable \\
Right: IV & Right: Severe & & & \\
III & Moderate & Duplication of pelvis and ureter with stenosis & Yes & Favorable \\
& & at the ureter end and ureterocele & & No \\
II & Severe & Duplication of pelvis & No & - \\
II & Moderate & & No & - \\
II & Moderate & Stenosis at the right ureteropelvic junction & No & - \\
II & Moderate & Stenosis at the left ureteropelvic junction & & - \\
\hline
\end{tabular}

SFU, Society for Fetal Urology; APD, anterior-posterior diameter; -, no related data.

independently associated with the occurrence of HY regression, which implied that these two grading systems may be used to predict post-partum HY regression.

The predominant treatment for persistent fetal HY is surgery and the prognosis is favorable (24). In spite of the availability of valuable approaches for outcome evaluation of fetal HY, including the SFU and APD grading systems (15), controversy remains regarding the post-partum management on the determination of surgery $(25,26)$. To date, certain parameters have been identified as indicators for the requirement of surgery, including ureteropelvic junction obstruction (27), the renal parenchyma to hydronephrosis area ratio (28) and cortical transit time (29). The present study also assessed the association of the SFU and APD grades with the surgery rate.
The Kaplan-Meier curves indicated that those patients with high SFU or APD grades had a higher surgery rate than those with low SFU or APD grades. The results of the two grading systems were proved to be independent predictors for the performance of surgery, indicating that they may serve as a guide for the decision to perform surgery.

In conclusion, the majority of cases of fetal HY spontaneously resolve after birth, and patients with pathological HY usually have high SFU and APD grades. Stenosis at the ureteropelvic junction was identified as a leading cause of pathological HY. The SFU and APD grades may be used as reliable predictors of the outcome of fetal HY, and may serve as independent indicators for the occurrence of $\mathrm{HY}$ regression and post-partum surgery. 


\section{Acknowledgements}

Not applicable.

\section{Funding}

This work was supported by the Wenzhou Science and Technology Project (grant. no. Y20180224).

\section{Availability of data and materials}

The datasets used and analyzed during the present study are available from the corresponding author on reasonable request.

\section{Authors' contributions}

DZ, YZ and PL designed this study, performed the APD and SFU grading, analyzed the data and wrote the manuscript. XS, $\mathrm{XC}, \mathrm{BY}$ and TL recruited the patients, collected the ultrasound results and analyzed the clinical data of the patients. YC, MY, LL and LM analyzed the association of SFU and APD grades with HY regression and surgery rate. All authors read and approved the final manuscript.

\section{Ethics approval and consent to participate}

The protocols of the present study were approved by the Ethics Committee of the Second Affiliated Hospital of Wenzhou Medical University (Wenzhou, China) and written informed consent for the use of ultrasonic data and clinical characteristics was obtained from each participant.

\section{Patient consent for publication}

Not applicable.

\section{Competing interests}

The authors declare that they have no competing interests.

\section{References}

1. Zampieri N, Zamboni C, Ottolenghi A and Camoglio FS: Unilateral hydronephrosis due to ureteropelvic junction obstruction in children: Long term follow-up. Minerva Urol Nefrol 61: 325-329, 2009

2. Herndon CD: The role of ultrasound in predicting surgical intervention for prenatal hydronephrosis. J Urol 187: 1535-1536, 2012

3. Plevani C, Locatelli A, Paterlini G, Ghidini A, Tagliabue P, Pezzullo JC and Vergani P: Fetal hydronephrosis: Natural history and risk factors for postnatal surgery. J Perinat Med 42: 385-391, 2014.

4. Delaney C: Antenatal hydronephrosis: Trends and management. Urol Nurs 25: 173-174, 179-183, 2005.

5. Braga LH, McGrath M, Farrokhyar F, Jegatheeswaran K and Lorenzo AJ: Society for fetal urology classification vs urinary tract dilation grading system for prognostication in prenatal hydronephrosis: A time to resolution analysis. J Urol 199: 1615-1621, 2018.

6. Gordon ZN, McLeod DJ, Ching CB, Herz DB, Bates DG, Becknell B and Alpert SA: Uroepithelial thickening improves detection of vesicoureteral reflux in infants with prenatal hydronephrosis. J Pediatr Urol 12: 257.e1-7, 2016.
7. Chevalier RL: Congenital urinary tract obstruction: The long view. Adv Chronic Kidney Dis 22: 312-319, 2015.

8. Zhan X, Tao G, Cheng L, Liu F, Li H and Liu S: Ultrasound score: A new method to differentiate fetal physiological and pathological hydronephrosis. Eur J Obstet Gynecol Reprod Biol 151: 26-32, 2010.

9. Liu DB, Armstrong WR III and Maizels M: Hydronephrosis: Prenatal and postnatal evaluation and management. Clin Perinatol 41: 661-678, 2014.

10. Oliveira EA, Oliveira MC and Mak RH: Evaluation and management of hydronephrosis in the neonate. Curr Opin Pediatr 28: 195-201, 2016.

11. Rao PK and Palmer JS: Prenatal and postnatal management of hydronephrosis. ScientificWorldJournal 9: 606-614, 2009.

12. Vemulakonda V, Yiee J and Wilcox DT: Prenatal hydronephrosis: Postnatal evaluation and management. Curr Urol Rep 15: 430, 2014.

13. Dos Santos J, Parekh RS, Piscione TD, Hassouna T, Figueroa V, Gonima P, Vargas I, Farhat W and Rosenblum ND: A new grading system for the management of antenatal hydronephrosis. Clin J Am Soc Nephrol 10: 1783-1790, 2015.

14. Zhang L, Liu C, Li Y, Sun C and Li X: Determination of the need for surgical intervention in infants diagnosed with fetal hydronephrosis in china. Med Sci Monit 22: 4210-4217, 2016.

15. Chalmers DJ, Meyers ML, Brodie KE, Palmer C and Campbell JB: Inter-rater reliability of the APD, SFU and UTD grading systems in fetal sonography and MRI. J Pediatr Urol 12: 305.e1-305.e5, 2016.

16. Zee RS, Herbst KW, Kim C, McKenna PH, Bentley T, Cooper CS and Herndon CD: Urinary tract infections in children with prenatal hydronephrosis: A risk assessment from the society for fetal urology hydronephrosis registry. J Pediatr Urol 12: 261.el-7, 2016.

17. Renda R: Renal outcome of congenital anomalies of the kidney and urinary tract system: A single-center retrospective study. Minerva Urol Nefrol 70: 218-225, 2018.

18. Woodward M and Frank D: Postnatal management of antenatal Hydronephrosis. BJU Int 89: 149-156, 2002.

19. Anjum M, Moorani KN, Sameen I, Mustufa MA and Kulsoom S: Functional and structural abnormalities of the kidney and urinary tract in severely malnourished children-A hospital based study. Pak J Med Sci 32: 1135-1140, 2016.

20. Rianthavorn P and Limwattana S: Diagnostic accuracy of neonatal kidney ultrasound in children having antenatal hydronephrosis without ureter and bladder abnormalities. World J Urol 33: 1645-1650, 2015.

21. Arger PH, Coleman BG, Mintz MC, Snyder HP, Camardese T, Arenson RL, Gabbe SG and Aquino L: Routine fetal genitourinary tract screening. Radiology 156: 485-489, 1985.

22. Wong DC, Anderson PA, Macken M and Jackson JR: Congenital hydronephrosis who requires intervention? Can J Urol 6: 812-818, 1999.

23. Swords KA and Peters CA: Neonatal and early infancy management of prenatally detected hydronephrosis. Arch Dis Child Fetal Neonatal Ed 100: F460-F464, 2015.

24. Al-Mashhadi A, Nevéus T, Stenberg A, Karanikas B, Persson AE, Carlström M and Wåhlin N: Surgical treatment reduces blood pressure in children with unilateral congenital hydronephrosis. J Pediatr Urol 11: 91.e1-6, 2015.

25. Gn MZ, Malik A, Hart LA, Mukherjee A and Reese AC: Hydronephrosis secondary to an ectopic decidual reaction in the urinary bladder. Urology 106: e11-e12, 2017.

26. Dy GW, Ellison JS, Fu BC, Holt SK, Gore JL and Merguerian PA: Variable resource utilization in the prenatal and postnatal management of isolated hydronephrosis. Urology 108: 155-160, 2017.

27. Arora S, Yadav P, Kumar M, Singh SK, Sureka SK, Mittal V and Ansari MS: Predictors for the need of surgery in antenatally detected hydronephrosis due to UPJ obstruction-a prospective multivariate analysis. J Pediatr Urol 11: 248.e1-5, 2015.

28. Rickard M, Lorenzo AJ and Braga LH: Renal parenchyma to hydronephrosis area ratio (PHAR) as a predictor of future surgical intervention for infants with high-grade prenatal hydronephrosis. Urology 101: 85-89, 2017.

29. Harper L, Bourquard D, Grosos C, Abbo O, Ferdynus C, Michel JL, Dunand O and Sauvat F: Cortical transit time as a predictive marker of the need for surgery in children with pelvi-ureteric junction stenosis: Preliminary study. J Pediatr Urol 9: 1054-1058, 2013. 